\title{
The exponential pencil of conics
}

\author{
Lorenz Halbeisen $^{1}$ • Norbert Hungerbühler ${ }^{1}$
}

Received: 3 October 2017 / Accepted: 13 December 2017 / Published online: 21 December 2017 (C) The Author(s) 2017. This article is an open access publication

\begin{abstract}
The exponential pencil $G_{\lambda}:=G_{1}\left(G_{0}^{-1} G_{1}\right)^{\lambda-1}$, generated by two conics $G_{0}, G_{1}$, carries a rich geometric structure: It is closed under conjugation, it is compatible with duality and projective mappings, it is convergent for $\lambda \rightarrow \pm \infty$ or periodic, and it is connected in various ways with the linear pencil $g_{\lambda}=\lambda G_{1}+(1-\lambda) G_{0}$. The structure of the exponential pencil can be used to characterize the position of $G_{0}$ and $G_{1}$ relative to each other.
\end{abstract}

Keywords Pencil of conics · Poncelet's theorem · Conjugate conics

Mathematics Subject Classification 51A05 - 51A10 - 51A20

\section{Introduction}

The linear pencil $g_{\lambda}=\lambda G_{1}+(1-\lambda) G_{0}, \lambda \in \mathbb{R}$, of two circles or conics $G_{0}$ and $G_{1}$ is an extremely useful tool in the study of the geometry of circles and of conic sections, or, in higher dimensions, of quadrics. The linear pencil has a wide range of applications: for example, the circles of Apollonius (see Coxeter 1989), Gergonne's solution of Apollonius' Problem to construct a circle touching three given circles (see Coolidge 1971), Cayley's characterization of conics which carry Poncelet polygons (see Cayley 1854), or the classification of the relative position of two conics (see Petitjean 2010). But the linear pencil is not only a tool, it is also an interesting object in its own right with

Norbert Hungerbühler
norbert.hungerbuehler@math.ethz.ch
Lorenz Halbeisen
lorenz.halbeisen@math.ethz.ch
$1 \quad$ Department of Mathematics, ETH Zentrum, Rämistrasse 101, 8092 Zürich, Switzerland 
a rich geometry to study. However, the linear pencil lacks certain desirable properties: For example, it is not compatible with duality, i.e., the linear pencil of the dual of two conics is not the dual of the pencil of the two conics (see Sects. 2.1 and 3), and the linear pencil does, in general, not exist as real conics for all $\lambda \in \mathbb{R}$. In this article, we investigate the exponential pencil $G_{\lambda}=G_{1}\left(G_{0}^{-1} G_{1}\right)^{\lambda-1}$ of two conics $G_{0}$ and $G_{1}$. It turns out, that this pencil has a remarkable spectrum of geometric properties, which we study in Sect. 3. In Sect. 4 we classify the exponential pencils according to the relative position of the generating conics. But first, we start with some preliminary remarks to set the stage and to fix the notation.

\section{Preliminaries}

\subsection{Matrix powers}

Let $f: \mathbb{R} \rightarrow \mathbb{C}^{n \times n}$ be analytic such that

(a) $f(0)=\mathbb{I}$, where $\mathbb{I}$ is the identity matrix,

(b) $f(1)=A$,

(c) $f(x+y)=f(x) \cdot f(y)$ for all $x, y \in \mathbb{R}$.

In particular, we have $f(-x)=f(x)^{-1}$ for all $x \in \mathbb{R}$, and therefore $A$ is necessarily regular. Moreover, all matrices $f(x), f(y)$ commute. With the infinitesimal generator $F:=f^{\prime}(0)$, we may write $f(x)=e^{F x}$. In particular, $A=f(1)=e^{F}$, i.e., $F$ is a logarithm of $A$. The logarithm of a matrix is in general not unique. Nonetheless, (a)-(c) determine the values of $f(n)$ for all $n \in \mathbb{Z}$. It is convenient to write $f(x)=A^{x}$ for a function satisfying (a)-(c). However, we have to keep in mind that two different logarithms of $A$ define different functions $x \mapsto A^{x}$. In concrete cases, a function $A^{x}$ can be calculated by the binomial series

$$
A^{x}=(\mathbb{I}+(A-\mathbb{I}))^{x}=\sum_{k=0}^{\infty}\left(\begin{array}{l}
x \\
k
\end{array}\right)(A-\mathbb{I})^{k}
$$

whenever the series converges.

Let $f(x)=A^{x}$ be a solution of (a)-(c), and suppose the matrix $A$ is similar to the matrix $B$, i.e., $B=T^{-1} A T$. Then $g(x):=T^{-1} f(x) T$ is analytic, $g(0)=\mathbb{I}$, $g(1)=T^{-1} A T=B$, and $g(x+y)=g(x) \cdot g(y)$ for arbitrary $x, y \in \mathbb{R}$. Thus, $g(x)=B^{x}$. In this situation, the infinitesimal generators of $f$ and $g$ are similar: $g^{\prime}(0)=T^{-1} f^{\prime}(0) T$.

\subsection{Projective plane and conics}

We will work in the standard model of the real projective plane, i.e., we consider the set of points $\mathbb{P}=\mathbb{R}^{3} \backslash\{0\} / \sim$, where $x \sim y \in \mathbb{R}^{3} \backslash\{0\}$ are equivalent if $x=\lambda y$ for some $\lambda \in \mathbb{R}$. The set of lines is $\mathbb{B}=\mathbb{R}^{3} \backslash\{0\} / \sim$, where $g \sim h \in \mathbb{R}^{3} \backslash\{0\}$ are equivalent, if $g=\lambda h$ for some $\lambda \in \mathbb{R}$. We that say a point $[x]$ and a line $[g]$ are 
incident if $\langle x, g\rangle=0$, where we denoted equivalence classes by square brackets and the standard inner product in $\mathbb{R}^{3}$ by $\langle\cdot, \cdot\rangle$.

As usual, a line $[g]$ can be identified with the set of points which are incident with it. Vice versa, a point $[x]$ can be identified with the set of lines which pass through it. The affine plane $\mathbb{R}^{2}$ is embedded in the present model of the projective plane by the map

$$
\left(\begin{array}{l}
x_{1} \\
x_{2}
\end{array}\right) \mapsto\left[\left(\begin{array}{c}
x_{1} \\
x_{2} \\
1
\end{array}\right)\right]
$$

The projective general linear group $\operatorname{PGL}(3, \mathbb{R})$ consists of equivalence classes $[A]$ of regular matrices $A \in \mathbb{R}^{3 \times 3}$ representing maps $\mathbb{P} \rightarrow \mathbb{P},[x] \mapsto[A x]$, where two matrices are equivalent, denoted $A_{1} \sim A_{2}$, if $A_{1}=\lambda A_{2}$ for some $\lambda \in \mathbb{R}$.

A conic in this model of the projective plane is an equivalence class of a regular, linear, selfadjoint map $A: \mathbb{R}^{3} \rightarrow \mathbb{R}^{3}$ with mixed signature, i.e., $A$ has eigenvalues of both signs. It is convenient to say a matrix $A$ is a conic, instead of $A$ is a representative of a conic. We may identify a conic by the set of points $[x]$ such that $\langle x, A x\rangle=0$, or by the set of lines $[g]$ for which $\left\langle A^{-1} g, g\right\rangle=0$ (see below). Notice that, in this interpretation, a conic cannot be empty: Since $A$ has positive and negative eigenvalues, there are points $[p],[q]$ with $\langle p, A p\rangle>0$ and $\langle q, A q\rangle<0$. Hence a continuity argument guarantees the existence of points $[x]$ satisfying $\langle x, A x\rangle=0$.

From now on, we will only distinguish in the notation between an equivalence class and a representative if necessary.

Fact 2.1 Let $x$ be a point on the conic A. Then the line Ax is tangent to the conic A with contact point $x$.

Proof We show that the line $A x$ meets the conic $A$ only in $x$. Suppose otherwise, that $y \nsim x$ is a point on the conic, i.e., $\langle y, A y\rangle=0$, and at the same time on the line $A x$, i.e., $\langle y, A x\rangle=0$. By assumption, we have $\langle x, A x\rangle=0$. Note, that $A x \nsim A y$ since $A$ is regular, and $\langle A y, x\rangle=0$ since $A$ is selfadjoint. Hence $x$ and $y$ both are perpendicular to the plane spanned by $A x$ and $A y$, which contradicts $y \nsim x$.

In other words, the set of tangents of a conic $A$ is the image of the points on the conic under the map $A$. And consequently, a line $g$ is a tangent of the conic iff $A^{-1} g$ is a point on the conic, i.e., if and only if $\left\langle A^{-1} g, g\right\rangle=0$.

Definition 2.2 If $P$ is a point, the line $A P$ is called its polar with respect to a conic $A$. If $g$ is a line, the point $A^{-1} g$ is called its pole with respect to the conic $A$.

Obviously, the pole of the polar of a point $P$ is again $P$, and the polar of the pole of a line $g$ is again $g$. Moreover:

Fact 2.3 If the polar of a point $P$ with respect to a conic $A$ intersects the conic in a point $x$, then the tangent in $x$ passes through $P$.

Proof For $x$, we have $\langle x, A x\rangle=0$ since $x$ is a point on the conic, and $\langle x, A P\rangle=0$ since $x$ is a point on the polar of $P$. The tangent in $x$ is the line $A x$, and indeed, $P$ lies on this line, since $\langle P, A x\rangle=\langle A P, x\rangle=0$. 
The fundamental theorem in the theory of poles and polars is

Fact 2.4 (La Hire's Theorem) Let $g$ be a line and $P$ its pole with respect to a conic A. Then, for every point $x$ on $g$, the polar of $x$ passes through $P$. And vice versa: Let $P$ be a point and $g$ its polar with respect to a conic A. Then, for every line $h$ through $P$, the pole of $h$ lies on $g$.

Proof We prove the second statement, the first one is similar. The polar of $P$ is the line $g=A P$. A line $h$ through $P$ satisfies $\langle P, h\rangle=0$ and its pole is $Q=A^{-1} h$. We check, that $Q$ lies on $g$ : Indeed, $\langle Q, g\rangle=\left\langle A^{-1} h, A P\right\rangle=\left\langle A A^{-1} h, P\right\rangle=\langle h, P\rangle=0$.

The next fact can be viewed as a generalization of Fact 2.4:

Theorem 2.5 Let $A$ and $G$ be conics. Then, for every point $x$ on $G$, the polar $p$ of $x$ with respect to $A$ is tangent to the conic $H=A G^{-1} A$ in the point $x^{\prime}=A^{-1} G x$. Moreover, $x^{\prime}$ is the pole of the tangent $g=G x$ in $x$ with respect to $A$.

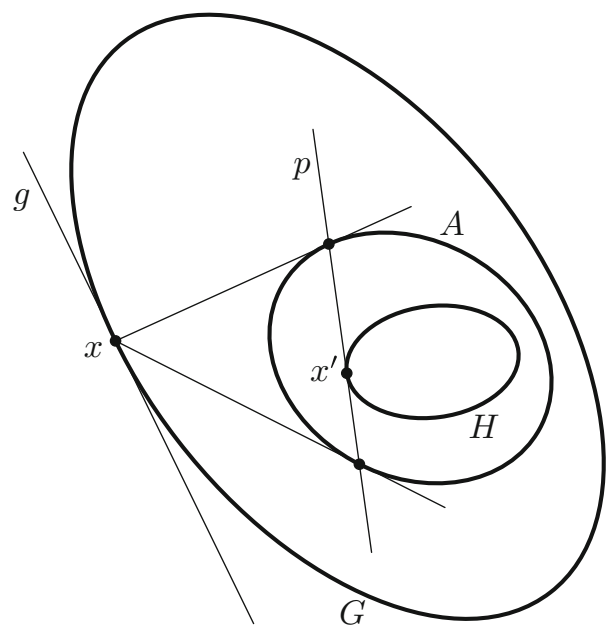

Proof It is clear that $H=A G^{-1} A$ is symmetric and regular, and by Sylvester's law of inertia, $H$ has mixed signature. The point $x$ on $G$ satisfies $\langle x, G x\rangle=0$. Its pole with respect to $A$ is the line $g=A x$. This line is tangent to $H$ iff $\left\langle H^{-1} g, g\right\rangle=0$. Indeed, $\left\langle H^{-1} g, g\right\rangle=\left\langle\left(A G^{-1} A\right)^{-1} A x, A x\right\rangle=\left\langle A^{-1} G x, A x\right\rangle=\langle G x, x\rangle=0$.

Since $\left\langle x^{\prime}, H x^{\prime}\right\rangle=\left\langle A^{-1} G x, A G^{-1} A A^{-1} G x\right\rangle=\langle G x, x\rangle=0$, the point $x^{\prime}=$ $A^{-1} G x$ lies on $H$. The tangent to $H$ in $x^{\prime}$ is $H x^{\prime}=A G^{-1} A A^{-1} G x=A x$ which is indeed the polar of $x$ with respect to $A$. The last statement in the theorem follows immediately.

Definition 2.6 The conic $H=A G^{-1} A$ is called the conjugate conic of $G$ with respect to $A$.

Recall that the dual of a point $P \in \mathbb{P}$ is the line $P \in \mathbb{B}$ and the dual of the line $g \in \mathbb{B}$ is the point $g \in \mathbb{P}$. In particular, $P$ and $g$ are incident if and only if their duals are 
incident. The dual lines of all points on a conic $A$ are tangent to the conic $A^{-1}$, and the dual points of all tangents of a conic $A$ are points on the conic $A^{-1}$. Therefore, $A^{-1}$ is called the dual conic of the conic $A$. We will denote the dual $A^{-1}$ of a conic $A$ by $A^{\prime}$.

The projective space $\mathbb{P}=\mathbb{R}^{3} \backslash\{0\} / \sim$ can also be represented as the unit sphere $S^{2} \subset \mathbb{R}^{3}$ with antipodal identification of points. Then, this space $\mathbb{S}$, endowed with the natural metric $d([x],[y])=\arcsin \|x \times y\|$, becomes a complete metric space with bounded metric. The set of closed sets in this space is a complete metric space with respect to the inherited Hausdorff metric. In particular, a conic $A$ given by

$$
\left\{x \in S^{2} \mid\langle x, A x\rangle=0\right\} / \sim
$$

is a compact set in $\mathbb{S}$. In this sense, we can consider the limit of a sequence of conics.

\section{The exponential pencil}

The linear pencil of two matrices $g_{0}, g_{1} \in \mathbb{C}^{n \times n}$ is given by

$$
g_{\lambda}:=\lambda g_{1}+(1-\lambda) g_{0}, \quad \lambda \in \mathbb{R} .
$$

This notation is consistent for the values $\lambda=0$ and $\lambda=1$. If $g_{0}$ and $g_{1}$ commute, exponentiation of the linear pencil gives

$$
G_{\lambda}:=e^{g_{\lambda}}=e^{\lambda g_{1}+(1-\lambda) g_{0}}=e^{g_{1}}\left(e^{-g_{0}} e^{g_{1}}\right)^{\lambda-1}=G_{1}\left(G_{0}^{-1} G_{1}\right)^{\lambda-1}
$$

where $G_{i}:=e^{g_{i}}$. The last expression in (1) makes sense also for non-commuting matrices and we may define an exponential pencil of two matrices $G_{0}, G_{1} \in \mathbb{C}^{n \times n}$ by

$$
G_{\lambda}:=G_{1}\left(G_{0}^{-1} G_{1}\right)^{\lambda-1}, \quad \lambda \in \mathbb{R},
$$

provided $\left(G_{0}^{-1} G_{1}\right)^{x}, x \in \mathbb{R}$, exists in the sense of Sect. 2.1. The notation $G_{\lambda}$ in (2) is consistent for the values $\lambda=0$ and $\lambda=1$. Notice that for regular matrices $G_{0}, G_{1}$, a unique discrete exponential pencil $G_{n}=G_{1}\left(G_{0}^{-1} G_{1}\right)^{n-1}$ for $n \in \mathbb{Z}$ exists. This general concept applies naturally to conics and we define:

Definition 3.1 Let $G_{0}, G_{1}$ be two conics. Then

$$
G_{\lambda}:=G_{1}\left(G_{0}^{-1} G_{1}\right)^{\lambda-1}, \quad \lambda \in \mathbb{R},
$$

is called an exponential pencil generated by $G_{0}$ and $G_{1}$ provided that all $G_{\lambda}$ are symmetric and real.

\section{Remarks}

(a) For an exponential pencil to exist, it is necessary and sufficient that $G_{0}^{-1} G_{1}$ has a real logarithm $F$ such that $G_{1} F$ is symmetric.

(b) In Sect. 4 we will see that the existence of an exponential pencil depends on the position of $G_{0}$ and $G_{1}$ relative to each other, and except for only one case, the exponential pencil is unique. 
(c) Each $G_{\lambda}$ in an exponential pencil generated by $G_{0}$ and $G_{1}$ is actually a conic: In contrast to the linear pencil, an exponential pencil of conics does not contain degenerate or complex conics. This is a consequence of the following Lemma.

Lemma 3.2 If $G_{\lambda}=G_{1}\left(G_{0}^{-1} G_{1}\right)^{\lambda-1}, \lambda \in \mathbb{R}$, is an exponential pencil of two conics $G_{0}, G_{1}$, then

(i) $\operatorname{det}\left(G_{\lambda}\right)=\operatorname{det}\left(G_{1}\right)^{\lambda} / \operatorname{det}\left(G_{0}\right)^{\lambda-1}$,

(ii) $G_{\lambda}$ has mixed signature for all $\lambda \in \mathbb{R}$.

Proof (i) Let $L$ be a logarithm of $G_{0}^{-1} G_{1}$. Then, we have

$$
\begin{aligned}
\operatorname{det} G_{\lambda} & =\operatorname{det} G_{1}\left(G_{0}^{-1} G_{1}\right)^{\lambda-1}=\operatorname{det} G_{1} \operatorname{det} e^{(\lambda-1) L}=\operatorname{det} G_{1} e^{\operatorname{trace}(\lambda-1) L} \\
& =\operatorname{det} G_{1}\left(e^{\operatorname{trace} L}\right)^{\lambda-1}=\operatorname{det} G_{1}\left(\operatorname{det} e^{L}\right)^{\lambda-1}=\operatorname{det} G_{1}\left(\frac{\operatorname{det} G_{1}}{\operatorname{det} G_{0}}\right)^{\lambda-1}
\end{aligned}
$$

(ii) Since $G_{\lambda}$ is symmetric, it has real eigenvalues which depend continuously on $\lambda$. Then, according to (i), the product of the eigenvalues cannot change sign and the signature of $G_{\lambda}$ remains constant.

The next Lemma will have immediate geometric consequences:

Lemma 3.3 If $G_{\lambda}, \lambda \in \mathbb{R}$, is an exponential pencil of $G_{0}, G_{1}$ and $\xi, \mu \in \mathbb{R}$, there holds

$$
G_{\mu} G_{\xi}^{-1} G_{\mu}=G_{2 \mu-\xi}
$$

Proof

$$
\begin{aligned}
G_{\mu} G_{\xi}^{-1} G_{\mu} & =G_{1}\left(G_{0}^{-1} G_{1}\right)^{\mu-1}\left(G_{1}\left(G_{0}^{-1} G_{1}\right)^{\xi-1}\right)^{-1} G_{1}\left(G_{0}^{-1} G_{1}\right)^{\mu-1} \\
& =G_{1}\left(G_{0}^{-1} G_{1}\right)^{\mu-1}\left(G_{0}^{-1} G_{1}\right)^{1-\xi} G_{1}^{-1} G_{1}\left(G_{0}^{-1} G_{1}\right)^{\mu-1} \\
& =G_{1}\left(G_{0}^{-1} G_{1}\right)^{2 \mu-\xi-1}=G_{2 \mu-\xi} .
\end{aligned}
$$

In view of Theorem 2.5 and Definition 2.6, we get as an immediate consequence of Lemma 3.3:

Theorem 3.4 An exponential pencil $G_{\lambda}, \lambda \in \mathbb{R}$, of two conics is closed under conjugation: The conjugate of $G_{\xi}$ with respect to $G_{\mu}$ is $G_{2 \mu-\xi}$.

More generally, we have the following:

Lemma 3.5 If $G_{\lambda_{0}}$ and $G_{\lambda_{1}}$ belong to a pencil $G_{\lambda}=G_{1}\left(G_{0}^{-1} G_{1}\right)^{\lambda-1}$ generated by $G_{0}, G_{1}$, then $G_{\lambda_{0}}$ and $G_{\lambda_{1}}$ generate the same exponential pencil as $G_{0}$ and $G_{1}$. More precisely, we have

$$
G_{\lambda_{1}}\left(G_{\lambda_{0}}^{-1} G_{\lambda_{1}}\right)^{\lambda-1}=G_{1}\left(G_{0}^{-1} G_{1}\right)^{\lambda_{0}+\lambda\left(\lambda_{1}-\lambda_{0}\right)-1}=G_{\lambda_{0}+\lambda\left(\lambda_{1}-\lambda_{0}\right)} .
$$


In particular, the exponential pencil does not depend on the order of the defining conics $G_{0}$ and $G_{1}$.

Proof Let $f(x):=\left(G_{0}^{-1} G_{1}\right)^{x\left(\lambda_{1}-\lambda_{0}\right)}$ for $x \in \mathbb{R}$. Then $f(0)=\mathbb{I}$, and

$$
\begin{aligned}
f(1) & =\left(G_{0}^{-1} G_{1}\right)^{\lambda_{1}-\lambda_{0}}=\left(G_{0}^{-1} G_{1}\right)^{1-\lambda_{0}} G_{1}^{-1} G_{1}\left(G_{0}^{-1} G_{1}\right)^{\lambda_{1}-1} \\
& =\left(G_{1}\left(G_{0}^{-1} G_{1}\right)^{\lambda_{0}-1}\right)^{-1} G_{1}\left(G_{0}^{-1} G_{1}\right)^{\lambda_{1}-1}=G_{\lambda_{0}}^{-1} G_{\lambda_{1}}
\end{aligned}
$$

Moreover, $f(x+y)=f(x) f(y)$. Therefore, according to Sect. 2.1, we may write $f(x)=\left(G_{\lambda_{0}}^{-1} G_{\lambda_{1}}\right)^{x}$. We obtain

$$
G_{\lambda_{1}}\left(G_{\lambda_{0}}^{-1} G_{\lambda_{1}}\right)^{\lambda-1}=G_{\lambda_{1}} f(\lambda-1)=G_{1}\left(G_{0}^{-1} G_{1}\right)^{\lambda_{1}-1}\left(G_{0}^{-1} G_{1}\right)^{(\lambda-1)\left(\lambda_{1}-\lambda_{0}\right)}
$$

where we used the original definition of $f$ in the last equality. Now the claim follows immediately.

It turns out that exponential pencils behave well with respect to duality:

Theorem 3.6 Let $G_{0}$ and $G_{1}$ be conics and $G_{0}^{\prime}$ and $G_{1}^{\prime}$ their duals. Suppose $G_{0}$ and $G_{1}$ generate an exponential pencil $G_{\lambda}$. Then, the dual of $G_{\lambda}$ is an exponential pencil of $G_{0}^{\prime}$ and $G_{1}^{\prime}$. More precisely, for all $\lambda \in \mathbb{R}$ we have

$$
G_{1}^{\prime}\left(G_{0}^{\prime-1} G_{1}^{\prime}\right)^{\lambda-1}=\left(G_{1}\left(G_{0}^{-1} G_{1}\right)^{\lambda-1}\right)^{\prime}
$$

Observe that the linear pencil does not enjoy the corresponding property.

Proof Suppose $G_{\lambda}=G_{1}\left(G_{0}^{-1} G_{1}\right)^{\lambda-1}$ is an exponential pencil generated by $G_{0}$ and $G_{1}$. Then, for $x \in \mathbb{R}$, let $f(x):=G_{1-x} G_{1}^{-1 s}=G_{1}\left(G_{0}^{-1} G_{1}\right)^{-x} G_{1}^{-1}$. Observe that $f(0)=\mathbb{I}, f(1)=G_{0} G_{1}^{-1}$ and

$$
\begin{aligned}
f(x+y) & =G_{1}\left(G_{0}^{-1} G_{1}\right)^{-(x+y)} G_{1}^{-1}=\left(G_{1}\left(G_{0}^{-1} G_{1}\right)^{-x} G_{1}^{-1}\right)\left(G_{1}\left(G_{0}^{-1} G_{1}\right)^{-y} G_{1}^{-1}\right) \\
& =f(x) f(y)
\end{aligned}
$$

and therefore, according to Sect. 2.1, we may write $f(x)=\left(G_{0} G_{1}^{-1}\right)^{x}=\left(G_{0}^{\prime-1} G_{1}^{\prime}\right)^{x}$. We obtain

$$
\left(G_{1}\left(G_{0}^{-1} G_{1}\right)^{x}\right)^{-1}=G_{1}^{-1} f(x)=G_{1}^{\prime}\left(G_{0}^{\prime-1} G_{1}^{\prime}\right)^{x}
$$

and claim follows by replacing $x$ by $\lambda-1$.

The natural question is now to ask which conics $G_{0}, G_{1}$ generate an exponential pencil. To answer this question, we recall that two conics can lie in 8 different positions relative to each other (see Petitjean 2010): 


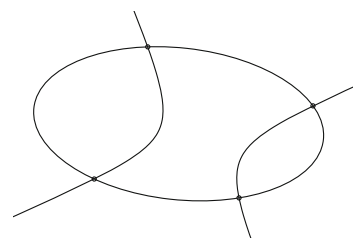

Case 1: four intersections

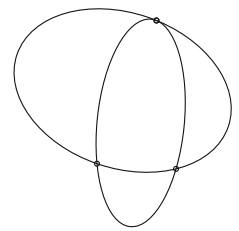

Case 4: two intersections, one 1st order contact

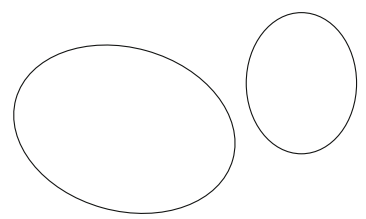

Case 2: no intersections

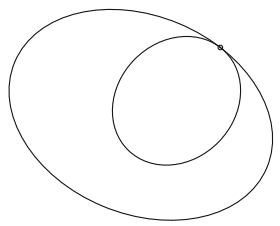

Case 5: one 1st order contact

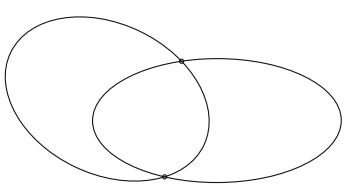

Case 3: two intersections
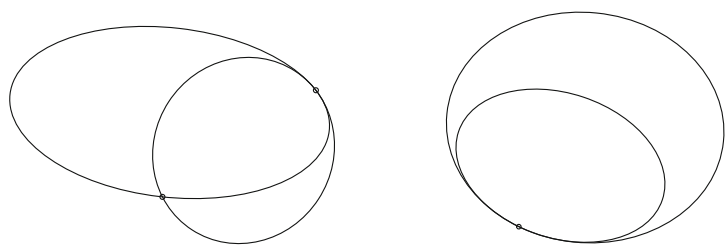

Case 7: one intersection, one Case 8: one 3rd order contact 2nd order contact

We now go case by case through the list and investigate the existence and the geometric properties of the resulting exponential conics. In particular, it will turn out that the exponential conic and the linear conic are quite closely related. We start with the important observation that the exponential pencil is projectively invariant:

Lemma 3.7 Let $S \in \mathbb{R}^{n \times n}$ be a regular matrix, inducing a projective map $\mathbb{P} \rightarrow$ $\mathbb{P}, x \mapsto S x$. Then the image under $S$ of an exponential pencil $G_{\lambda}=G_{1}\left(G_{0}^{-1} G_{1}\right)^{\lambda-1}$ of two conics $G_{0}, G_{1}$ is an exponential pencil of their images.

Proof For $T:=S^{-1}$, the images of the conics $G_{0}, G_{1}$ under $S$ are $\bar{G}_{0}:=T^{\top} G_{0} T$ and $\bar{G}_{1}:=T^{\top} G_{1} T$. We want to show that the image $\bar{G}_{\lambda}=T^{\top} G_{\lambda} T$ is an exponential pencil of $\bar{G}_{0}$ and $\bar{G}_{1}$. We start by definig $f(x):=T^{-1}\left(G_{0}^{-1} G_{1}\right)^{x} T$ for $x \in \mathbb{R}$. We have $f(0)=\mathbb{I}, f(1)=\bar{G}_{0}^{-1} \bar{G}_{1}$ and

$$
\begin{aligned}
f(x+y) & =T^{-1}\left(G_{0}^{-1} G_{1}\right)^{x+y} T=\left(T^{-1}\left(G_{0}^{-1} G_{1}\right)^{x} T\right)\left(T^{-1}\left(G_{0}^{-1} G_{1}\right)^{y} T\right) \\
& =f(x) f(y)
\end{aligned}
$$

and therefore, according to Sect. 2.1, we may write $f(x)=\left(\bar{G}_{0}^{-1} \bar{G}_{1}\right)^{x}$. We obtain

$$
T^{\top} G_{x+1} T=T^{\top} G_{1}\left(G_{0}^{-1} G_{1}\right)^{x} T=\bar{G}_{1} f(x)=\bar{G}_{1}\left(\bar{G}_{0}^{-1} \bar{G}_{1}\right)^{x}
$$

and claim follows by replacing $x$ by $\lambda-1$. 
The investigation of the exponential pencils in all the Cases 1-8 listed above can now be reduced to a canonical form in each case.

\section{Classification of the exponential pencils}

The two figures below show the exponential pencil of two conics $G_{0}, G_{1}$ (bold) in two cases. On the left, the geometry seems rather gentle, on the right quite complex.
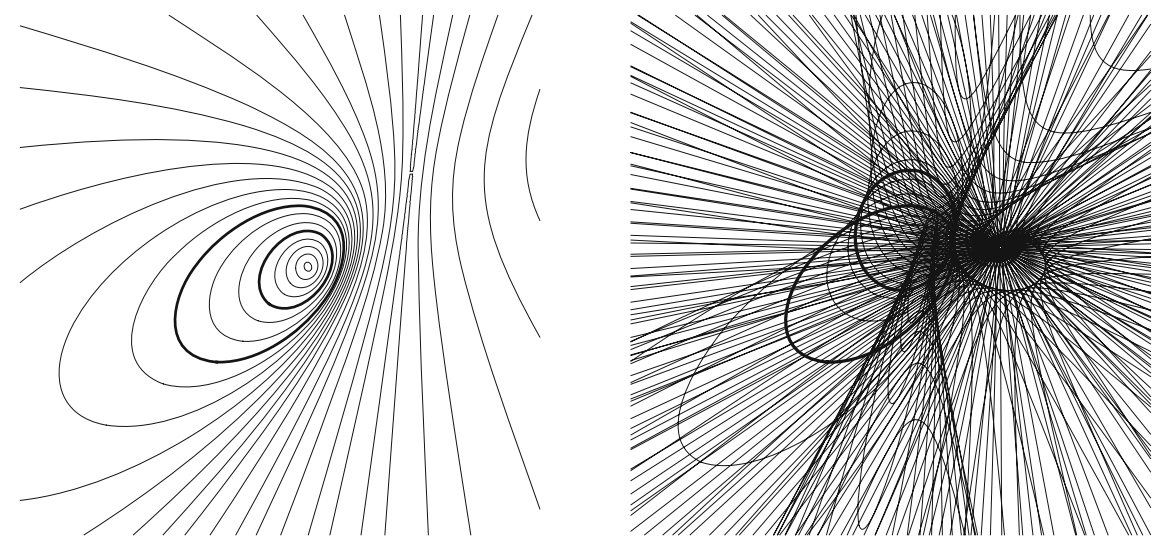

In this section, we investigate the exponential pencil of two conics in each of the possible cases of their relative position. It turns out that the geometric behavior of the exponential pencil is characteristic for each case.

Theorem 4.1 (Case 1) Let $G_{0}, G_{1}$ be two conics with four intersection points. Then, they generate an exponential conic $G_{\lambda}=G_{1}\left(G_{0}^{-1} G_{1}\right)^{\lambda-1}$ iff the common interior of $G_{1}$ and $G_{0}$ is connected. In this case, the exponential pencil is unique. $G_{\lambda}$ converges for $\lambda \rightarrow \pm \infty$ to a line $\ell^{ \pm}$. The family $G_{\lambda}$ has an envelope $E$ with asymptotes $\ell^{ \pm}$. Through every exterior point of $E$ (i.e., points with four tangents to $E$ ), except for the points on $\ell^{ \pm}$, there pass exactly two members of the exponential pencil $G_{\lambda}$. Each $G_{\lambda}$ touches a member of the linear pencil $g_{\lambda}=\lambda G_{1}+(1-\lambda) G_{0}$ in two first order contact points.

Proof After applying a suitable projective map, we may assume that

$$
G_{0}=\left(\begin{array}{ccc}
1 & 0 & 0 \\
0 & 1 & 0 \\
0 & 0 & -1
\end{array}\right), \quad G_{1}=\left(\begin{array}{ccc}
a^{2} & 0 & 0 \\
0 & \pm b^{2} & 0 \\
0 & 0 & -1
\end{array}\right)
$$

where $a>1>b>0$ or $b>1>a>0$ for the positive sign, and $a>1$ for the negative sign (see Halbeisen and Hungerbühler 2017). Let

$$
A:=G_{0}^{-1} G_{1}=\left(\begin{array}{ccc}
a^{2} & 0 & 0 \\
0 & \pm b^{2} & 0 \\
0 & 0 & 1
\end{array}\right)
$$


then every solution $X$ of $e^{X}=A$ leads to an exponential pencil $G_{\lambda}=G_{1} e^{(\lambda-1) X}$ of $G_{0}$ and $G_{1}$, provided $G_{\lambda}$ is a real symmetric matrix for all $\lambda \in \mathbb{R}$. In particular, $h(x):=e^{x X}$ must be real for all $x \in \mathbb{R}$. But then $h^{\prime}(0)=X$ must be real. We can therefore concentrate on real solutions of $e^{X}=A$. According to Culver (1966, Theorem 1), such a real solution exists only for the positive sign in $A$. This corresponds to the case, where the common interior of $G_{0}$ and $G_{1}$ is connected. Then, the solution of $e^{X}=A$ is unique, according to Culver (1966, Theorem 2), and we obtain a unique exponential pencil given by

$$
G_{\lambda}=G_{1}\left(G_{0}^{-1} G_{1}\right)^{\lambda-1}=\left(\begin{array}{ccc}
a^{2 \lambda} & 0 & 0 \\
0 & b^{2 \lambda} & 0 \\
0 & 0 & -1
\end{array}\right)
$$

The envelope $E$ is obtained by eliminating $\lambda$ from $\frac{\partial}{\partial \lambda}\left\langle x, G_{\lambda} x\right\rangle=0$ and $\left\langle x, G_{\lambda} x\right\rangle=0$. One finds

$$
\left(x_{1}^{2}\right)^{\log b}\left(x_{3}^{2}\right)^{\log a}|\log a|^{\log a}\left|\log \frac{a}{b}\right|^{\log b}=\left(x_{2}^{2}\right)^{\log a}\left(x_{3}^{2}\right)^{\log b}|\log b|^{\log b}\left|\log \frac{a}{b}\right|^{\log a} .
$$

The figure shows in the affine plane $x_{3}=1$ the pencil generated by the unit circle $G_{0}$ and an ellipse $G_{1}$ (both bold), together with the asymptotic lines $\ell^{ \pm}$(red) and the envelope $E$ (blue).

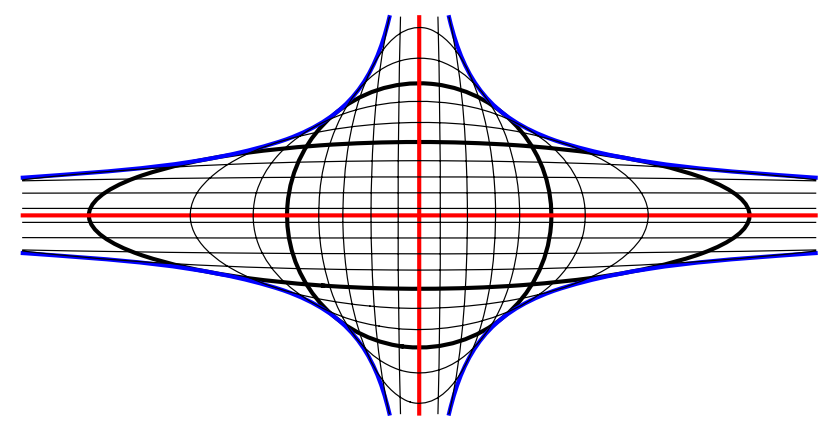

Theorem 4.2 (Case 2) Let $G_{0}, G_{1}$ be two disjoint conics. Then they generate an exponential pencil $G_{\lambda}=G_{1}\left(G_{0}^{-1} G_{1}\right)^{\lambda-1}$ iff $G_{1}$ is in the interior of $G_{0}$ or vice versa, in which case the exponential pencil is unique. $G_{\lambda}$ converge for $\lambda \rightarrow \pm \infty$ to a point (which coincides with a limit point of the linear pencil $g_{\lambda}=\lambda G_{1}+(1-\lambda) G_{0}$ ), and a line (which contains the second limit point of the linear pencil). Each $G_{\lambda}$ touches two members of the linear pencil $g_{\lambda}=\lambda G_{1}+(1-\lambda) G_{0}$ in two first order contact points, or, if $G_{0}, G_{1}$ are projectively equivalent to concentric circles, each $G_{\lambda}$ belongs to the linear pencil. 
Proof Since $G_{0}, G_{1}$ are disjoint, there exist coordinates for which both conics are diagonal [see for example Pesonen (1956) or Hong et al. (1986)]: W.l.o.g.

$$
G_{0}=\left(\begin{array}{ccc}
1 & 0 & 0 \\
0 & 1 & 0 \\
0 & 0 & -1
\end{array}\right), \quad G_{1}=\left(\begin{array}{ccc}
a^{2} & 0 & 0 \\
0 & \pm b^{2} & 0 \\
0 & 0 & -1
\end{array}\right)
$$

where $1>a, b>0$ or $a, b>1$ in case of the positive sign, and $1>a>0, b>0$ in case of the negative sign. Then,

$$
A:=G_{0}^{-1} G_{1}=\left(\begin{array}{ccc}
a^{2} & 0 & 0 \\
0 & \pm b^{2} & 0 \\
0 & 0 & 1
\end{array}\right)
$$

As in Case 1, an exponential pencil can only exist for the positive sign in $A$. This corresponds to the case where $G_{0}$ is in the interior of $G_{1}$ or vice versa. Now, we have to consider two cases:

Case $2 a . a \neq b$ : Then, by the same reasoning as in Case 1 , the exponential pencil $G_{\lambda}$ is unique and given by (3). The figure on the left shows, in the plane $x_{3}=1$, the exponential pencil generated by the unit circle $G_{0}$ and an ellipse $G_{1}$ inside of $G_{0}$ (both bold). The limit as $\lambda \rightarrow \infty$ is the center (red), and as $\lambda \rightarrow-\infty$ the ideal line. It is instructive to look at the same configuration on the sphere (figure on the right, limit point and limit ideal line in red).
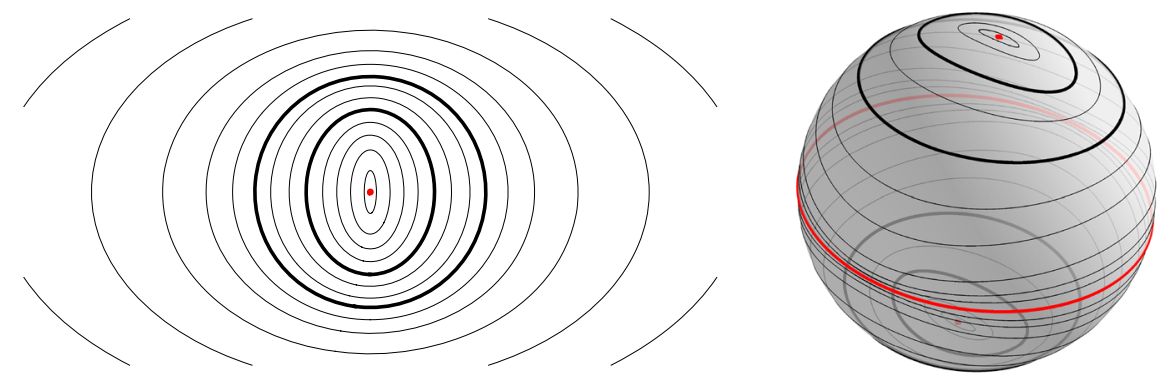

Case 2b. $a=b$ : In this case we have

$$
A:=G_{0}^{-1} G_{1}=\left(\begin{array}{ccc}
a^{2} & 0 & 0 \\
0 & a^{2} & 0 \\
0 & 0 & 1
\end{array}\right)
$$

and according to Culver (1966, Theorem 2, and Corollary), there is a continuum of real solutions of $e^{X_{\mu}}=A$. So, there is a chance that the exponential pencil is not unique. From Gantmacher $(1998, \S 8)$ we infer that all matrices

$$
X_{\mu}=\left(\begin{array}{ccc}
\log a^{2} & 0 & 0 \\
0 & \log a^{2} & 0 \\
0 & 0 & 0
\end{array}\right)+K\left(\begin{array}{ccc}
2 n \pi i & 0 & 0 \\
& 2 m \pi i & 0 \\
0 & 0 & 0
\end{array}\right) K^{-1}
$$


where $m, n$ are integers and $K$ is an arbitrary regular matrix of the form

$$
K=\left(\begin{array}{ccc}
k_{11} & k_{12} & 0 \\
k_{21} & k_{22} & 0 \\
0 & 0 & k_{33}
\end{array}\right)
$$

are logarithms of $A$, and there are no other logarithms. Then $e^{(\lambda-1) X_{\mu}}$ has the same block structure as $K$. Now, in our case, we need that $G_{\lambda}=G_{1} e^{(\lambda-1) X_{\mu}}$ is real and symmetric. But this implies that $G_{1}^{-1} G_{\lambda}=e^{(\lambda-1) X_{\mu}}$ is real and symmetric for all $\lambda$. Then the derivative of this with respect to $\lambda$ at $\lambda=1$ gives that $X_{\mu}$ must be real and symmetric. Then for each $k \in \mathbb{N}, e^{X_{\mu} / 2^{k}}$ is also symmetric and real, and positive definite, because $e^{X_{\mu} / 2^{k}}=e^{X_{\mu} / 2^{k+1}} e^{X_{\mu} / 2^{k+1}}$. Recall that repeated roots $A^{1 / 2^{k}}$ of $A$ which are real, symmetric and positive definite, are unique. This means, that the values of $e^{X_{\mu} / 2^{k}}$ agree for all integers $k$. Therefore, the infinitesimal generators $X_{\mu}$ must actually agree. In other words, there is only one real symmetric logarithm $X$ of $A$, and the exponential pencil is given by (3), i.e. a family of concentric circles. Alternatively, the uniqueness can be seen directly from (4) by imposing symmetry and real valuedness of $X_{\mu}$.

Theorem 4.3 (Case 3 ) Let $G_{0}, G_{1}$ be two conics with two intersectctions. Then they generate a countable family of exponential pencils $G_{\lambda}=G_{1}\left(G_{0}^{-1} G_{1}\right)^{\lambda-1}$. Such a pencil is either periodic with a conic as envelope, or periodically expanding covering the plane infinitely often, with a local envelope which has a singular point S. For integer values of $\lambda$, the corresponding conics of all exponential pencils agree.

Proof After applying a suitable projective map, we may assume that

$$
G_{0}=\left(\begin{array}{ccc}
1 & 0 & 0 \\
0 & 1 & 0 \\
0 & 0 & -1
\end{array}\right), \quad G_{1}=\left(\begin{array}{ccc}
1 & 0 & -a \\
0 & 1 & 0 \\
-a & 0 & a^{2}-r^{2}
\end{array}\right), \quad a>0
$$

(see Halbeisen and Hungerbühler 2017). Geometrically, $G_{1}$ represents a circle of radius $r>0$ in the plane $x_{3}=1$ with center in $(a, 0)$ which intersects the unit circle $G_{0}$, centered in $(0,0)$, in two real points. I.e., $-1<a-r<1$ and $1<a+r$, which implies that $\kappa:=(1-a+r)(1+a-r)(a+r-1)(a+r+1)>0$ because all four factors are strictly positive. We now use a translation $T$, a swap of axis $P$, a scaling $L$, and a rotation $R$, namely

$$
\begin{aligned}
T & =\left(\begin{array}{lll}
1 & 0 & \tau \\
0 & 1 & 0 \\
0 & 0 & 1
\end{array}\right), \quad P=\left(\begin{array}{lll}
1 & 0 & 0 \\
0 & 0 & 1 \\
0 & 1 & 0
\end{array}\right), \\
L & =\left(\begin{array}{ccc}
\ell & 0 & 0 \\
0 & 1 / \ell & 0 \\
0 & 0 & 1
\end{array}\right), \quad R=\left(\begin{array}{ccc}
c & -\sqrt{1-c^{2}} & 0 \\
\sqrt{1-c^{2}} & c & 0 \\
0 & 0 & 1
\end{array}\right),
\end{aligned}
$$


with the following values

$$
\begin{aligned}
\tau & =\frac{1+a^{2}-r^{2}}{2 a}, \\
\ell & =\frac{\sqrt[4]{\kappa}}{\sqrt{2 a}}, \\
c & =\frac{1}{2} \sqrt{2-\frac{\sqrt{\kappa}}{a}} .
\end{aligned}
$$

Notice that $4 a^{2}-\kappa=\left(1+a^{2}-r^{2}\right)^{2} \geq 0$ and hence the radicand $2-\frac{\sqrt{\kappa}}{a} \geq 0$ in $c$. For $U=T P L R$ this leads to the following representation of the conics:

$$
U^{\top} G_{0} U=\left(\begin{array}{ccc}
-1 & 0 & 0 \\
0 & 1 & 0 \\
0 & 0 & 1
\end{array}\right), U^{\top} G_{1} U=\left(\begin{array}{ccc}
\left(a^{2}-r^{2}-1\right) / 2 & \sqrt{\kappa} / 2 & 0 \\
\sqrt{\kappa} / 2 & \left(r^{2}-a^{2}+1\right) / 2 & 0 \\
0 & 0 & 1
\end{array}\right)
$$

In the plane $x_{3}=1$ these are rotated hyperbolas centered at $(0,0,1)$, and we denote them again by $G_{0}$ and $G_{1}$. Then, $G_{0}^{-1} G_{1}$ has the form

$$
A:=G_{0}^{-1} G_{1}=\left(\begin{array}{ccc}
r \cos \phi_{k} & -r \sin \phi_{k} & 0 \\
r \sin \phi_{k} & r \cos \phi_{k} & 0 \\
0 & 0 & 1
\end{array}\right)
$$

for $\phi_{k}=2 k \pi+\arccos \frac{1-a^{2}+r^{2}}{2 r}$, where $k$ is an arbitrary integer. Notice, that $-2 r<$ $1-a^{2}+r^{2}<2 r$, again because the factors of $\kappa$ are strictly positive, and hence the values $\phi_{k}$ are real. Here, according to Gantmacher (1998, §8), we find the following solutions $X$ of $A=e^{X_{k}}$ :

$$
X_{k}=\left(\begin{array}{ccc}
\log r & -\phi_{k} & 0 \\
\phi_{k} & \log r & 0 \\
0 & 0 & 0
\end{array}\right)
$$

Therefore, we get

$$
e^{(\lambda-1) X_{k}}=\left(G_{0}^{-1} G_{1}\right)^{\lambda-1}=\left(\begin{array}{ccc}
r^{\lambda-1} \cos (\lambda-1) \phi_{k} & -r^{\lambda-1} \sin (\lambda-1) \phi_{k} & 0 \\
r^{\lambda-1} \sin (\lambda-1) \phi_{k} & r^{\lambda-1} \cos (\lambda-1) \phi_{k} & 0 \\
0 & 0 & 1
\end{array}\right)
$$

and finally

$$
G_{\lambda}=G_{1}\left(G_{0}^{-1} G_{1}\right)^{\lambda-1}=\left(\begin{array}{ccc}
-r^{\lambda} \cos \lambda \phi_{k} & r^{\lambda} \sin \lambda \phi_{k} & 0 \\
r^{\lambda} \sin \lambda \phi_{k} & r^{\lambda} \cos \lambda \phi_{k} & 0 \\
0 & 0 & 1
\end{array}\right)
$$


For $r=1$ (and only in this case), the resulting exponential pencil is periodic with period $2 \pi / \phi_{k}$. Hence, in the plane $x_{3}=1, G_{\lambda}$ are rectangular hyperbolas, rotating around the origin with constant angular velocity $\phi_{k}$. For $r \neq 1$, the rectangular hyperbolas are rotating with constant angular velocity $\phi_{k}$ and at the same time exponentially shrinking ( $r>1)$ or expanding $(0<r<1)$ with factor $r^{\lambda}$. The figures below show the two cases: $G_{0}$ and $G_{1}$ are bold, the envelope is blue, the singular point $S$ is red.
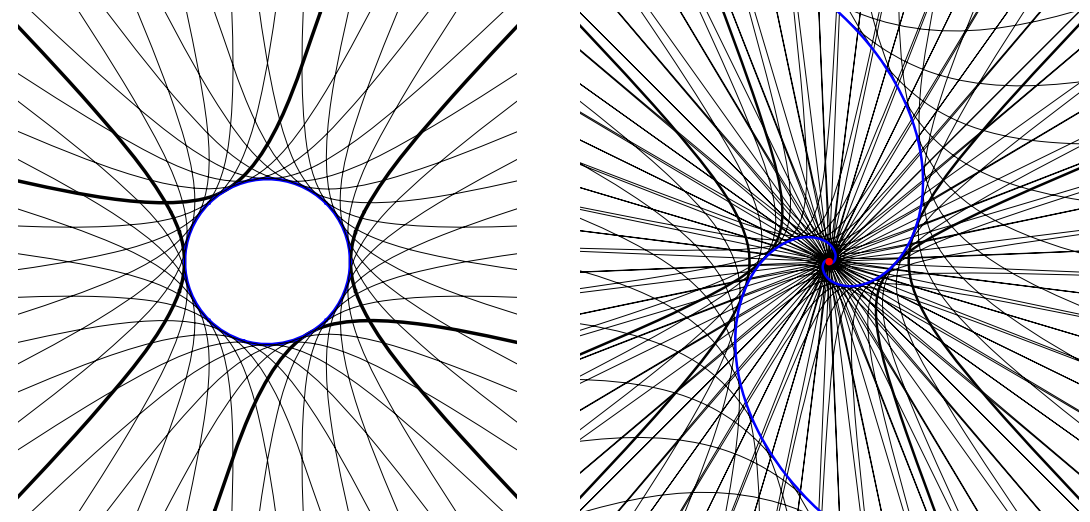

Remark The case when $r=1$ (i.e., when the resulting exponential pencil is periodic), was studied with respect to Poncelet's Theorem in Halbeisen and Hungerbühler (2016) and Halbeisen and Hungerbühler (2017).

Theorem 4.4 (Case 4) Let $G_{0}, G_{1}$ be two conics with two intersections and one first order contact. Then they generate an exponential pencil $G_{\lambda}=G_{1}\left(G_{0}^{-1} G_{1}\right)^{\lambda-1}$ iff the contact point of $G_{1}$ and $G_{0}$ lies on the boundary of their common interior. Then the exponential pencil is unique. Each $G_{\lambda}$ touches a member of the linear pencil $g_{\lambda}=\lambda G_{1}+(1-\lambda) G_{0}$ in two first order contact points. For $\lambda \rightarrow \pm \infty, G_{\lambda}$ converges to the tangent in the contact point, and to a line trough the contact point, respectively. The family $G_{\lambda}$ has an envelope $E$.

Proof After applying a suitable projective map, we may assume that

$$
G_{0}=\left(\begin{array}{ccc}
1 & 0 & 0 \\
0 & 1 & 0 \\
0 & 0 & -1
\end{array}\right), \quad G_{1}=\left(\begin{array}{ccc}
\mu+1 & 0 & -\mu \\
0 & 1-\mu & 0 \\
-\mu & 0 & \mu-1
\end{array}\right), \quad \mu \neq 1, \mu \neq 0
$$

(see Halbeisen and Hungerbühler (2017)). Then,

$$
A:=G_{0}^{-1} G_{1}=\left(\begin{array}{ccc}
\mu+1 & 0 & -\mu \\
0 & 1-\mu & 0 \\
\mu & 0 & 1-\mu
\end{array}\right)
$$


With

$$
T=\left(\begin{array}{ccc}
1 & 1 / \mu & 0 \\
0 & 0 & 1 \\
1 & 0 & 0
\end{array}\right), \quad J=\left(\begin{array}{ccc}
1 & 1 & 0 \\
0 & 1 & 0 \\
0 & 0 & 1-\mu
\end{array}\right)=\mathbb{I}+\underbrace{\left(\begin{array}{ccc}
0 & 1 & 0 \\
0 & 0 & 0 \\
0 & 0 & -\mu
\end{array}\right)}_{=: \alpha}
$$

we get $G_{0}^{-1} G_{1}=\mathbb{I}+T \alpha T^{-1}$. As in the proof of Case $2 \mathrm{~b}$, we are only interested in real logarithms of $A$. By Culver (1966, Theorem 1), the real logarithm of $A$ exists iff $\mu<1$. This corresponds to the situation where the contact point sits on the boundary of the common interior of $G_{0}$ and $G_{1}$. By Culver (1966, Theorem 2), the real logarithm is unique. By the binomic series we get

$$
\begin{aligned}
\left(G_{0}^{-1} G_{1}\right)^{x} & =\left(\mathbb{I}+T \alpha T^{-1}\right)^{x}=T \sum_{k=0}^{\infty}\left(\begin{array}{l}
x \\
k
\end{array}\right) \alpha^{k} T^{-1} \\
& =\left(\begin{array}{ccc}
1+\mu x & 0 & -\mu x \\
0 & (1-\mu)^{x} & 0 \\
\mu x & 0 & 1-\mu x
\end{array}\right),
\end{aligned}
$$

and finally the exponential pencil

$$
G_{\lambda}=G_{1}\left(G_{0}^{-1} G_{1}\right)^{\lambda-1}=\left(\begin{array}{ccc}
1+\lambda \mu & 0 & -\lambda \mu \\
0 & (1-\mu)^{\lambda} & 0 \\
-\lambda \mu & 0 & \lambda \mu-1
\end{array}\right)
$$

Notice that the binomial series converges only for $|\mu|<1$. But the expression we got for $\left(G_{0}^{-1} G_{1}\right)^{x}$ satisfies the properties of Sect. 2.1 and therefore the result for $G_{\lambda}$ is correct for arbitrary $\mu<1, \mu \neq 0$. The conics $G_{\lambda}$ are symmetric to the line $(0,1,0)^{\top}$ and touch $G_{0}, G_{1}$ in their contact point. The envelope $E$ is obtained by eliminating $\lambda$ from $\frac{\partial}{\partial \lambda}\left\langle x, G_{\lambda} x\right\rangle=0$ and $\left\langle x, G_{\lambda} x\right\rangle=0$. In the plane $x_{3}=1$ one finds

$$
\left(1+x_{1}\right) \ln (1-\mu)=\left(1-x_{1}\right) \mu\left(\ln \left(-\frac{\mu\left(x_{1}-1\right)^{2}}{x_{2}^{2} \ln (1-\mu)}\right)-1\right) .
$$

The figure shows, in the plane $x_{3}=1$, the pencil generated by the unit circle $G_{0}$ and an ellipse $G_{1}$ (both bold) together with the limiting lines (red) and the envelope $E$ (blue). 


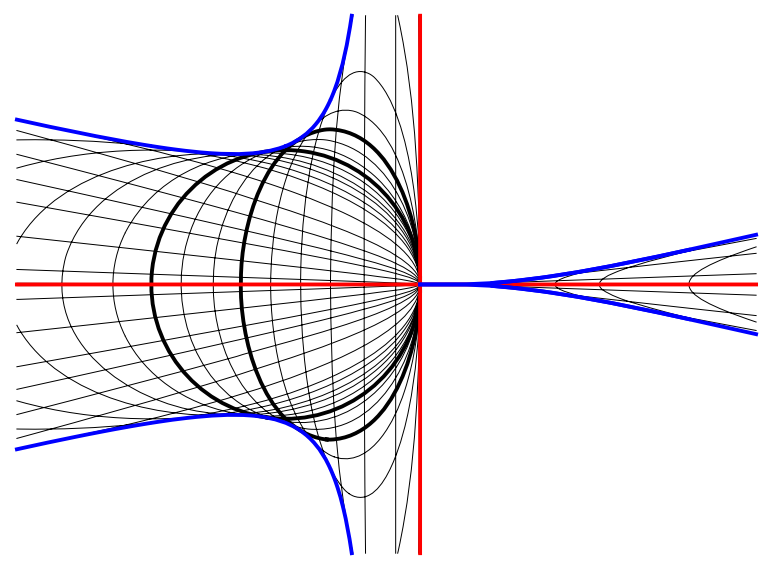

Theorem 4.5 (Case 5) Let $G_{0}, G_{1}$ be two conics with one first order contact point $C$. Then, they generate an exponential pencil $G_{\lambda}=G_{1}\left(G_{0}^{-1} G_{1}\right)^{\lambda-1}$ iff $G_{1}$ lies inside of $G_{0}$ or vice versa. This exponential pencil is unique. The family $G_{\lambda}$ together with the tangent in $C$ forms a foliation of $\mathbb{P} \backslash\{C\}$. Each $G_{\lambda}$ touches a member of the linear pencil $g_{\lambda}=\lambda G_{1}+(1-\lambda) G_{0}$ in two first order contact points. If $G_{1}$ is inside of $G_{0}$, then $G_{\lambda}$ converges to $C$ for $\lambda \rightarrow \infty$, and to the tangent in $C$ for $\lambda \rightarrow-\infty$. If $G_{0}$ lies inside of $G_{1}$ it is the other way round.

Proof After applying a suitable projective map, we may assume that

$$
G_{0}=\left(\begin{array}{ccc}
1 & 0 & 0 \\
0 & 1 & 0 \\
0 & 0 & -1
\end{array}\right), \quad G_{1}=\left(\begin{array}{ccc}
1 & 0 & -a \\
0 & 1 & 0 \\
-a & 0 & 2 a-1
\end{array}\right), \quad a \neq 1, a \neq 0
$$

(see Halbeisen and Hungerbühler 2017), i.e., $G_{0}$ is a unit circle centered in $(0,0,1)^{\top}$ and $G_{1}$ a circle with center $(a, 0,1)^{\top}$ which touches $G_{0}$ in $(1,0,1)^{\top}$. Then,

$$
A:=G_{0}^{-1} G_{1}=\left(\begin{array}{ccc}
1 & 0 & -a \\
0 & 1 & 0 \\
a & 0 & 1-2 a
\end{array}\right)
$$

With

$$
T=\left(\begin{array}{ccc}
0 & 0 & 1 / a \\
1 & 0 & 0 \\
0 & 1 & 0
\end{array}\right), \quad J=\left(\begin{array}{ccc}
1 & 0 & 0 \\
0 & 1-a & 1 \\
0 & 0 & 1-a
\end{array}\right)=\mathbb{I}+\underbrace{\left(\begin{array}{ccc}
0 & 0 & 0 \\
0 & -a & 1 \\
0 & 0 & -a
\end{array}\right)}_{=: \alpha}
$$

we get $G_{0}^{-1} G_{1}=\mathbb{I}+T \alpha T^{-1}$. As in Case 4, the real logarithm of $A$ exists, and is unique, iff $1>a$. This corresponds to the case where $G_{0}$ is inside $G_{1}$ or vice versa. 
Then, by the binomic series, we get

$$
\begin{aligned}
\left(G_{0}^{-1} G_{1}\right)^{x} & =\left(\mathbb{I}+T \alpha T^{-1}\right)^{x}=T \sum_{k=0}^{\infty}\left(\begin{array}{l}
x \\
k
\end{array}\right) \alpha^{k} T^{-1} \\
& =\left(\begin{array}{ccc}
(1-a)^{x-1}(1+a(x-1)) & 0 & -(1-a)^{x-1} a x \\
\mu & 1 & 0 \\
(1-a)^{x-1} a x & 0 & (1-a)^{x-1}(1-a(x+1))
\end{array}\right),
\end{aligned}
$$

and finally the exponential pencil

$$
G_{\lambda}=G_{1}\left(G_{0}^{-1} G_{1}\right)^{\lambda-1}=\left(\begin{array}{ccc}
(1-a)^{\lambda-1}(1+a(\lambda-1)) & 0 & -(1-a)^{\lambda-1} a \lambda \\
0 & 1 & 0 \\
-(1-a)^{\lambda-1} a \lambda & 0 & (1-a)^{\lambda-1}(a(\lambda+1)-1)
\end{array}\right)
$$

Notice that the binomial series converges only for $|a|<1$. However, the expression we obtained for $\left(G_{0}^{-1} G_{1}\right)^{x}$ satisfies the properties of Sect. 2.1 and therefore, the result for $G_{\lambda}$ is correct for arbitrary $a<1, a \neq 0$. The conics $G_{\lambda}$ are symmetric to the line $(0,1,0)^{\top}$ and touch $G_{0}, G_{1}$ in $C$. The figure shows, in the plane $x_{3}=1$, the pencil generated by the unit circle $G_{0}$ and a circle $G_{1}$ inside of $G_{0}$ (both bold), together with the tangent in the contact point (red).

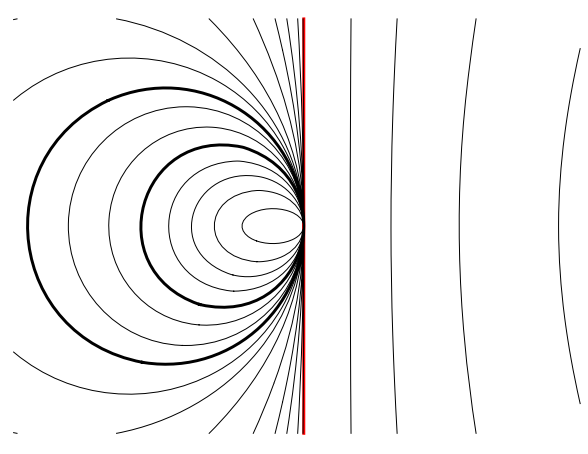

Theorem 4.6 (Case 6) Let $G_{0}, G_{1}$ be two conics with two first order contact points $C_{0}, C_{1}$. Then they generate an exponential pencil $G_{\lambda}=G_{1}\left(G_{0}^{-1} G_{1}\right)^{\lambda-1}$ iff $G_{0}$ lies inside of $G_{1}$ or vice versa. This exponential pencil is unique, and each conic $G_{\lambda}$ is a member of the linear pencil $g_{\lambda}=\lambda G_{1}+(1-\lambda) G_{0}$. If $G_{1}$ is inside of $G_{0}$, then $G_{\lambda}$ and $g_{\lambda}$ have the same limit for $\lambda \rightarrow \infty$, and for $\lambda \rightarrow-\infty$ the limit of $G_{\lambda}$ consists of the tangents in $C_{0}$ and $C_{1}$. If $G_{0}$ is inside of $G_{1}$ it is the other way round.

The proof will actually give some more information. 
Proof After applying a suitable projective map, we may assume that

$$
G_{0}=\left(\begin{array}{ccc}
1 & 0 & 0 \\
0 & 1 & 0 \\
0 & 0 & -1
\end{array}\right), \quad G_{1}=\left(\begin{array}{ccc}
1 & 0 & 0 \\
0 & 1-\mu & 0 \\
0 & 0 & -1
\end{array}\right), \quad \mu \neq 0, \mu \neq 1
$$

(see Halbeisen and Hungerbühler 2017). Then,

$$
A:=G_{0}^{-1} G_{1}=\left(\begin{array}{ccc}
1 & 0 & 0 \\
& 1-\mu & 0 \\
0 & 0 & 1
\end{array}\right)
$$

Like in Case 2, $A$ has only one symmetric, real logarithm if $\mu<1$. This inequality is equivalent to the fact that one conic lies inside the other, and we get

$$
\left(G_{0}^{-1} G_{1}\right)^{x}=\left(\begin{array}{ccc}
1 & 0 & 0 \\
& (1-\mu)^{x} & 0 \\
0 & 0 & 1
\end{array}\right)
$$

In this case, we obtain as exponential pencil

$$
G_{1}\left(G_{0}^{-1} G_{1}\right)^{\lambda-1}=\left(\begin{array}{ccc}
1 & 0 & 0 \\
& (1-\mu)^{\lambda} & 0 \\
0 & 0 & -1
\end{array}\right)=g_{\left(1-(1-\mu)^{\lambda}\right) / \mu}
$$

The figure shows the pencil generated by the unit circle $G_{0}$ and an ellipse $G_{1}$ (both bold) together with the limits (red).

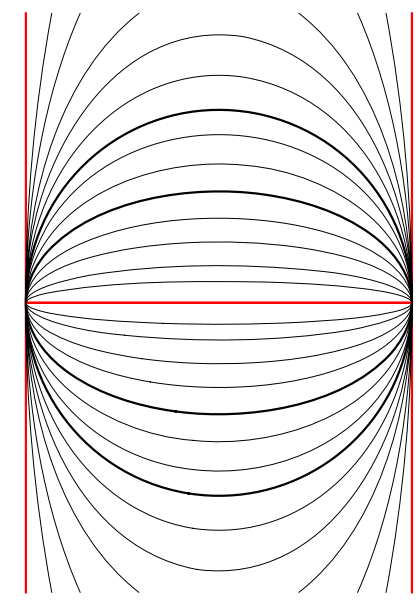

Theorem 4.7 (Case 7) Let $G_{0}, G_{1}$ be two conics with one intersection and one second order contact. Then, they generate a unique exponential pencil $G_{\lambda}=$ 
$G_{1}\left(G_{0}^{-1} G_{1}\right)^{\lambda-1}$. The family $G_{\lambda}$ has a conic $E$ as envelope. $E$ belongs to the linear pencil of $G_{2}-3 G_{0}-6 G_{1}$ and the double line joining the intersection point and the second order contact point of $G_{0}$ and $G_{1}$. Through every exterior point of $E$, except for the tangent in the contact point of $G_{0}$ and $G_{1}$, there pass exactly two members of the exponential pencil $G_{\lambda}$.

Proof After applying a suitable projective map, we may assume that

$$
G_{0}=\left(\begin{array}{ccc}
1 & 0 & 0 \\
0 & 1 & 0 \\
0 & 0 & -1
\end{array}\right), \quad G_{1}=\left(\begin{array}{ccc}
1 & -\mu & 0 \\
-\mu & 1 & \mu \\
0 & \mu & -1
\end{array}\right), \quad \mu \neq 0
$$

(see Halbeisen and Hungerbühler 2017). Then,

$$
A:=G_{0}^{-1} G_{1}=\left(\begin{array}{ccc}
1 & -\mu & 0 \\
-\mu & 1 & \mu \\
0 & -\mu & 1
\end{array}\right)
$$

With

$$
T=\left(\begin{array}{ccc}
1 & 0 & 1 / \mu^{2} \\
0 & -1 / \mu & 0 \\
1 & 0 & 0
\end{array}\right), \quad J=\left(\begin{array}{ccc}
1 & 1 & 0 \\
0 & 1 & 1 \\
0 & 0 & 1
\end{array}\right)=\mathbb{I}+\underbrace{\left(\begin{array}{ccc}
0 & 1 & 0 \\
0 & 0 & 1 \\
0 & 0 & 0
\end{array}\right)}_{=: \alpha}
$$

we get $G_{0}^{-1} G_{1}=\mathbb{I}+T \alpha T^{-1}$. By Culver (1966, Theorem 2), $A$ has a unique real logarithm, and we can use the binomic series (which, in this case, consists of only three terms), to obtain

$$
\begin{aligned}
\left(G_{0}^{-1} G_{1}\right)^{x} & =\left(\mathbb{I}+T \alpha T^{-1}\right)^{x}=T \sum_{k=0}^{\infty}\left(\begin{array}{l}
x \\
k
\end{array}\right) \alpha^{k} T^{-1} \\
& =\left(\begin{array}{ccc}
1+x(x-1) \mu^{2} / 2-x \mu & x(1-x) \mu^{2} / 2 \\
-x \mu & 1 & x \mu \\
x(x-1) \mu^{2} / 2 & -x \mu & 1+x(1-x) \mu^{2} / 2
\end{array}\right),
\end{aligned}
$$

and finally the exponential pencil

$$
G_{\lambda}=G_{1}\left(G_{0}^{-1} G_{1}\right)^{\lambda-1}=\left(\begin{array}{ccc}
1+\lambda(\lambda-1) \mu^{2} / 2-\lambda \mu & \lambda(1-\lambda) \mu^{2} / 2 \\
-\lambda \mu & 1 & \mu \lambda \\
\lambda(1-\lambda) \mu^{2} / 2 & \mu \lambda & \mu^{2} \lambda(\lambda-1) / 2-1
\end{array}\right) .
$$


The envelope $E$ is obtained by eliminating $\lambda$ from $\frac{\partial}{\partial \lambda}\left\langle x, G_{\lambda} x\right\rangle=0$ and $\left\langle x, G_{\lambda} x\right\rangle=0$. One finds the conic

$$
E=\left(\begin{array}{ccc}
\mu^{2}-8 & 4 \mu & -\mu^{2} \\
4 \mu & 8 & -4 \mu \\
-\mu^{2} & -4 \mu & 8+\mu^{2}
\end{array}\right)=G_{2}-3 G_{0}-6 G_{1}+16\left(\begin{array}{ccc}
0 & 0 & 0 \\
0 & 1 & 0 \\
0 & 0 & 0
\end{array}\right)
$$

It is then a simple calculation to check, that $\left\langle x, G_{\lambda} x\right\rangle=0$ has exactly two solutions $\lambda$ whenever $x$ is in the interior of $E$ and away from the tangent in the contact point of $G_{0}$ and $G_{1}$. The figure shows in the plane $x_{3}=1$ the pencil generated by the unit circle $G_{0}$ and an ellipse $G_{1}$ (both bold) together with the envelope $E$ (blue).

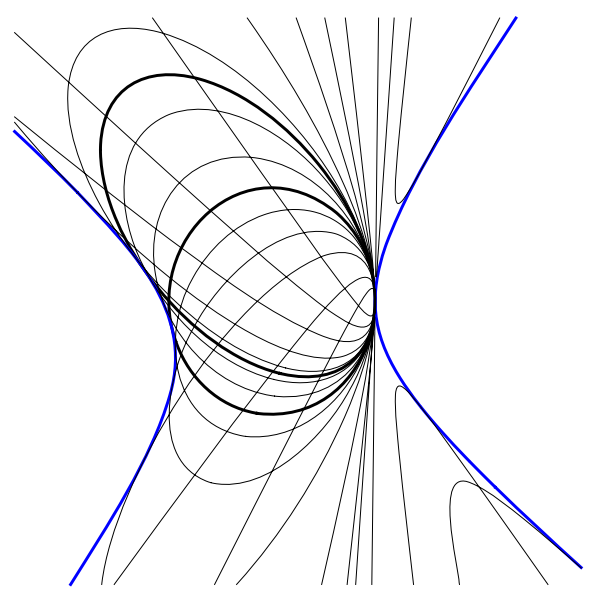

Theorem 4.8 (Case 8) Let $G_{0}, G_{2}$ be two conics with one third order contact point $C$. Then they generate a unique exponential pencil $G_{\lambda}=G_{1}\left(G_{0}^{-1} G_{1}\right)^{\lambda-1}$ which coincides with the linear pencil $g_{\lambda}=\lambda G_{1}+(1-\lambda) G_{0}$. The pencil $G_{\lambda}$ together with the tangent $t$ in $C$ yields a foliation of the projective space outside $C$. For $\lambda \rightarrow \pm \infty$, $G_{\lambda}$ converges to $t$ and $C$ respectively.

Proof After applying a suitable projective map, we may assume that

$$
G_{0}=\left(\begin{array}{ccc}
1 & 0 & 0 \\
0 & 1 & 0 \\
0 & 0 & -1
\end{array}\right), \quad G_{1}=\left(\begin{array}{ccc}
1 & 0 & 0 \\
0 & \mu+1 & -\mu \\
0 & -\mu & \mu-1
\end{array}\right), \quad \mu \neq 0
$$

(see Halbeisen and Hungerbühler 2017). Then,

$$
A:=G_{0}^{-1} G_{1}=\left(\begin{array}{ccc}
1 & 0 & 0 \\
0 & \mu+1 & -\mu \\
0 & \mu & 1-\mu
\end{array}\right)
$$


With

$$
T=\left(\begin{array}{ccc}
0 & 0 & 1 \\
1 & 1 / \mu & 0 \\
1 & 0 & 0
\end{array}\right), \quad J=\left(\begin{array}{ccc}
1 & 1 & 0 \\
0 & 1 & 0 \\
0 & 0 & 1
\end{array}\right)=\mathbb{I}+\underbrace{\left(\begin{array}{lll}
0 & 1 & 0 \\
0 & 0 & 0 \\
0 & 0 & 0
\end{array}\right)}_{=: \alpha}
$$

we get $G_{0}^{-1} G_{1}=\mathbb{I}+T \alpha T^{-1}$. Again, we have a unique real logarithm of $A$ and therefore, by the binomic series (which, in this case, consists of only two terms), we get

$$
\left(G_{0}^{-1} G_{1}\right)^{x}=\left(\mathbb{I}+T \alpha T^{-1}\right)^{x}=T \sum_{k=0}^{\infty}\left(\begin{array}{l}
x \\
k
\end{array}\right) \alpha^{k} T^{-1}=\left(\begin{array}{ccc}
1 & 0 & 0 \\
0 & 1+x \mu & -x \mu \\
0 & x \mu & 1-x \mu
\end{array}\right)
$$

and finally the exponential pencil

$$
G_{\lambda}=G_{1}\left(G_{0}^{-1} G_{1}\right)^{\lambda-1}=\left(\begin{array}{ccc}
1 & 0 & 0 \\
0 & \mu \lambda+1 & -\mu \lambda \\
0 & -\mu \lambda & \mu \lambda-1
\end{array}\right)=g_{\lambda}
$$

It is easy to check, that for every point $P \notin t$ there is exacly one $\lambda$ such that $\left\langle P, G_{\lambda} P\right\rangle=$ 0 The figure shows in the plane $x_{3}=1$ the pencil generated by the unit circle $G_{0}$ and a hyperbola $G_{1}$ (both bold) and the limits (red).

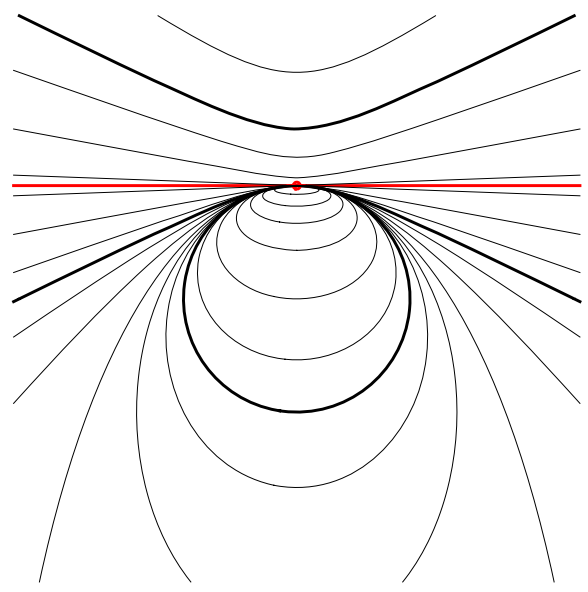

\section{A triangle center}

Starting with the circumcircle $G_{0}$ and the incircle $G_{1}$ of a triangle $\Delta_{0}=A_{0} B_{0} C_{0}$, we obtain a discrete chain of conjugate conics $G_{n}=G_{1}\left(G_{0}^{-1} G_{1}\right)^{n-1}$, for $n=0,1,2, \ldots$. Because of Theorem 2.5, the triangle $\Delta_{1}$ joining the contact points $A_{1}, B_{1}, C_{1}$ of the 
incircle of $\Delta_{0}$ is tangent to $G_{2}$. Iteration of this construction yields a sequence of triangles $\Delta_{n}$ (see figure below) having vertices on $G_{n}$ and sides tangent to $G_{n+1}$. The corresponding contact points on $G_{n+1}$ are the vertices of $\Delta_{n+1}$. This is a chain of dual Poncelet triangles in the sense of Halbeisen and Hungerbühler (2016).

According to Theorem 4.2, the linear and the exponental pencil of $G_{0}$ and $G_{1}$ have the same limit point. Hence, the sequence of triangles $\Delta_{n}$ converges together with the $G_{n}$ for $n \rightarrow \infty$ to the dilation center $X$ of $\Delta_{0}$ : This is Triangle Center $X(3513)$ in the Encyclopedia of Triangle Centers [5]. This center has hereby a new interpretation. The figure shows the situation for a triangle $\Delta_{0}$ (blue) and $G_{0}, G_{1}$ (bold) with the limit point $X$ (red).

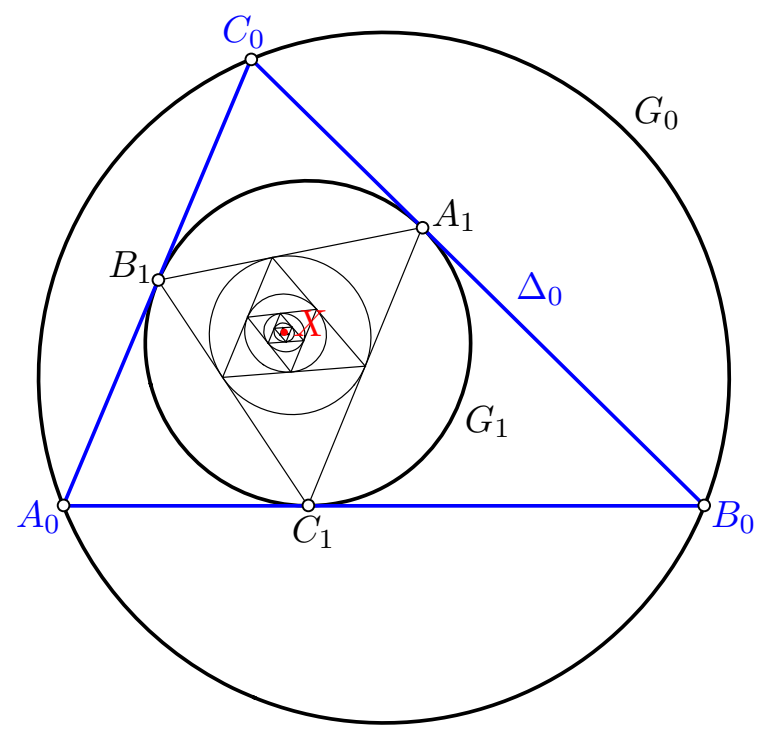

Since $\Delta_{0}$ is a Poncelet triangle for $G_{0}, G_{1}$, any other point $A_{0}^{\prime}$ on $G_{0}$ defines a triangle $\Delta_{0}^{\prime}$ with vertices $A_{0}^{\prime}, B_{0}^{\prime}, C_{0}^{\prime}$ on $G_{0}$ with incircle $G_{1}$. Each such triangle $\Delta_{0}^{\prime}$ generates a chain of dual Poncelet triangles with the same center $X$.

Acknowledgements We would like to thank the referee for his careful reading and the valuable suggestions which greatly helped to improve the article.

Open Access This article is distributed under the terms of the Creative Commons Attribution 4.0 International License (http://creativecommons.org/licenses/by/4.0/), which permits unrestricted use, distribution, and reproduction in any medium, provided you give appropriate credit to the original author(s) and the source, provide a link to the Creative Commons license, and indicate if changes were made.

\section{References}

Cayley, A.: Developments on the porism of the in-and-circumscribed polygon. Philos. Mag. 7, 339-345 (1854)

Coolidge, J.L.: A treatise on the circle and the sphere. Chelsea Publishing Co, Bronx (1971). (Reprint of the 1916 edition)

Coxeter, H.S.M.: Introduction to geometry. Wiley Classics Library. Wiley, New York (1989). (Reprint of the 1969 edition) 
Culver, W.J.: On the existence and uniqueness of the real logarithm of a matrix. Proc. Am. Math. Soc. 17, 1146-1151 (1966)

Clark K.: Encyclopedia of Triangle Centers. http://faculty.evansville.edu/ck6/encyclopedia/etcpart3.html. Accessed 18 Dec 2017

Gantmacher, F.R.: The theory of matrices. Vol. 1. AMS Chelsea Publishing, Providence (1998). (Translated from the Russian by K. A. Hirsch, Reprint of the 1959 translation)

Halbeisen, L., Hungerbühler, N.: Conjugate conics and closed chains of Poncelet polygons. Mitteilungen der Mathematischen Gesellschaft in Hamburg 36, 5-28 (2016)

Halbeisen, L., Hungerbühler, N.: Closed chains of conics carrying poncelet triangles. Beitr. Algebra Geom. 58(2), 277-302 (2017)

Hong, Y.P., Horn, R.A., Johnson, C.R.: On the reduction of pairs of Hermitian or symmetric matrices to diagonal form by congruence. Linear Algebra Appl. 73, 213-226 (1986)

Pesonen, E.: Über die Spektraldarstellung quadratischer Formen in linearen Räumen mit indefiniter Metrik. Ann. Acad. Sci. Fenn. Ser. A. I. 227, 31 (1956)

Petitjean, S.: Invariant-based characterization of the relative position of two projective conics. In: Nonlinear computational geometry, volume 151 of IMA Vol. Math. Appl., pp. 189-220. Springer, New York (2010) 